\title{
Liposome-encapsulation increases the bioavailability of midazolam on oral administration
}

\author{
Hiroshi Tanimura, DDS, PhD'1), Megumi Mori-Yoshioka, DDS, PhD'1), Yuka Honda- \\ Wakasugi, DDS, PhD2), Hitoshi Higuchi, DDS, PhD'1), Shigeru Maeda, DDS, PhD'1), \\ Takuya Miyawaki, DDS, $\mathrm{PhD}^{2)}$
}

1) Department of Dental Anesthesiology, Okayama University Hospital

2) Department of Dental Anesthesiology and Special Care Dentistry, Okayama University Graduate School of Medicine, Dentistry and Pharmaceutical Sciences

\section{Background}

The oral administration of midazolam has often been used for sedation in pediatric patients. However, its bioavailability is low on oral administration because it is metabolized in both the intestine and liver at a high rate. Liposomes have been developed as vesicles encapsulating various kinds of drug to serve as a medical drugdelivery system (Fig.1). We developed liposome-encapsulated midazolam for oral administration (Ref. 1). The purpose of the present study was to evaluate the bioavailability of liposomeencapsulated midazolam after oral administration in rabbits.

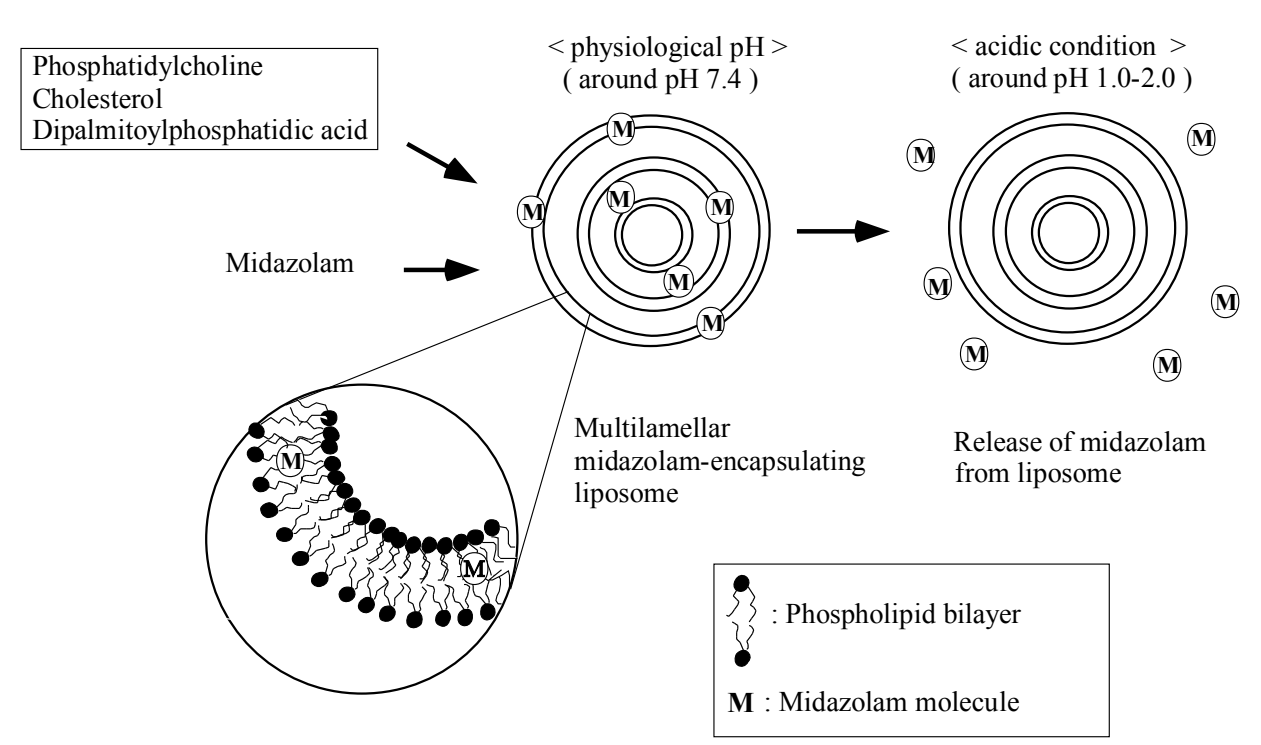

Fig.1 Liposome-encapsulated midazolam for oral administration

\section{Materials and Methods}

Liposome-encapsulated midazolam was produced from hydrogenated L-a-phosphatidylcholine, cholesterol, dipalmitoylphosphatidic acid, polyethylene glycol, and midazolam. A lipid film produced from the mixed materials was suspended and briefly sonicated in a water bath, resulting in the production of liposome-encapsulated midazolam (LE-midazolam) (Fig.2) . Furthermore, because smaller liposomes are expected to be efficiently absorbed from the intestine, we also produced miniaturized liposome-encapsulated midazolam (MLEmidazolam) using a sonic vibrator with ultrasonic waves (Fig. 3). After being approved by the Animal Care and Use Committee, we conducted experiments using 13 male New Zealand white rabbits (10-11 weeks old). LE-midazolam, MLE-midazolam, or midazolam solution was orally administered at the same dose of 2 $\mathrm{mg} / \mathrm{kg}$, and blood samples were collected until 4 hours after the administration. Blood midazolam concentrations of the samples were measured using HPLC, and analyzed using two-way ANOVA followed by Turkey's multiple comparisons test.

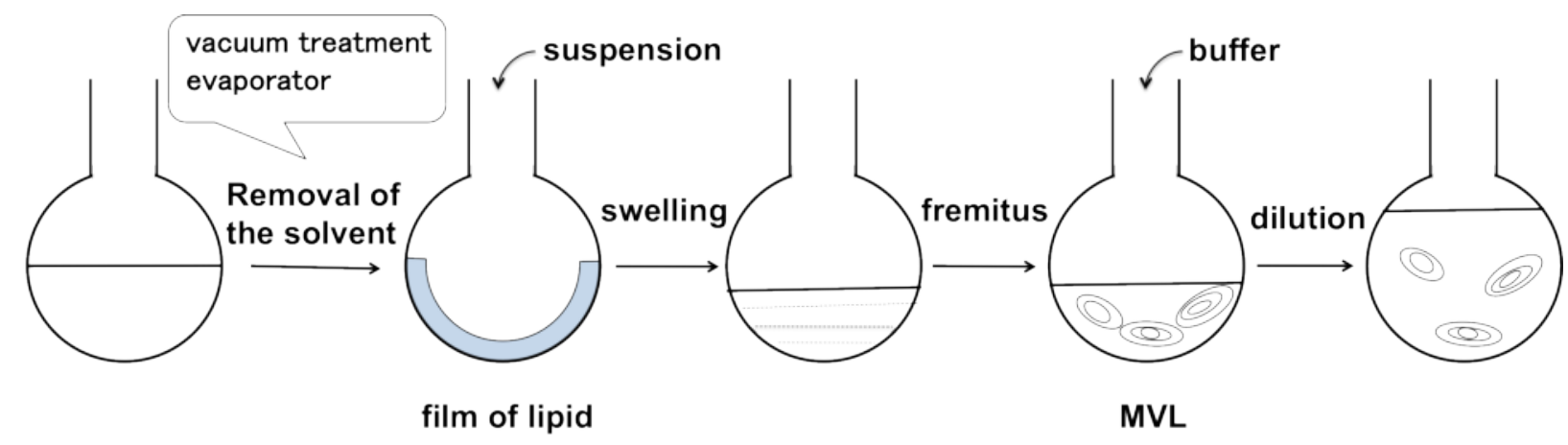

Fig. 2 Production of midazolam-encapsulating liposomes

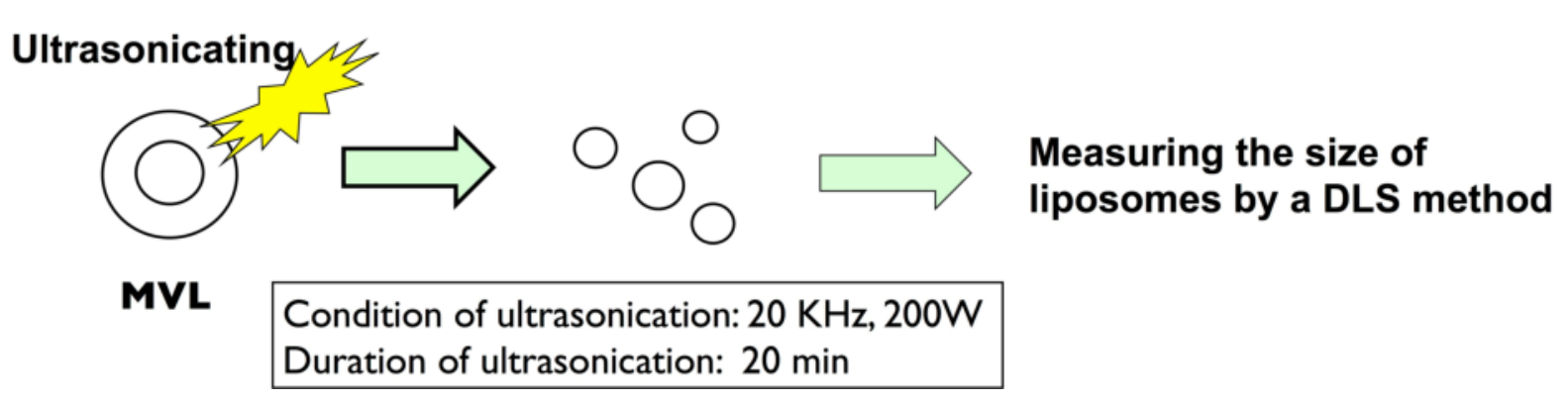

Fig. 3 Miniaturization of the midazolam-encapsulating liposomes

\section{Results and Discussion}

Blood midazolam concentrations in rabbits administered LEmidazolam and MLE-midazolam solutions were significantly higher than that in rabbits administered midazolam solution (Fig. 4). It is possible that the encapsulating liposomes inhibited the metabolism of midazolam and/or increased its absorption in the intestine.

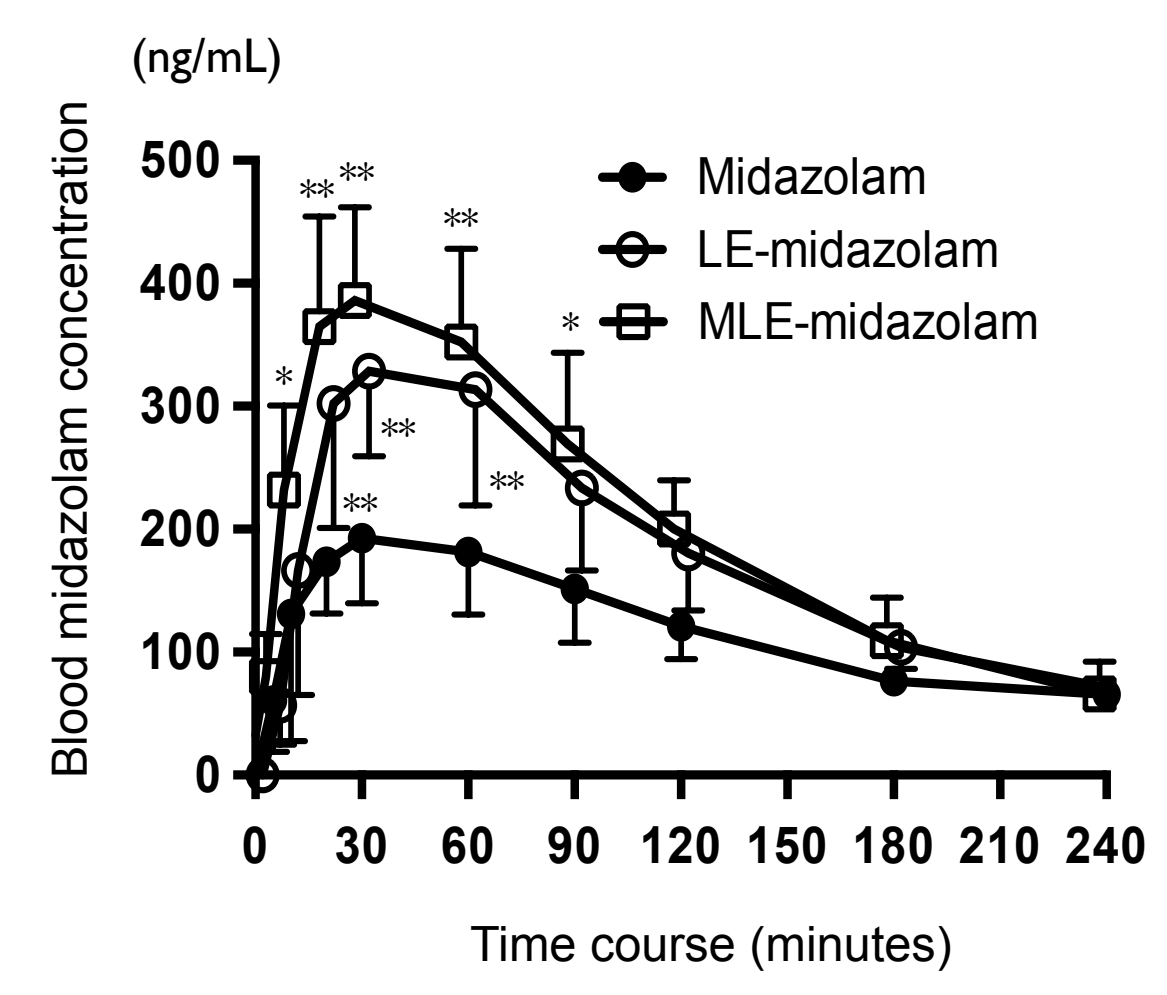

Fig. 4 Blood midazolam concentration after oral administration LE-midazolam: liposome-encapsulated midazolam solution MLE-midazolam: miniaturized liposome-encapsulated midazolam solution

${ }^{*} \mathrm{P}<0.05,{ }^{* *} \mathrm{P}<0.01$ vs. midazolam

\section{Conclusion}

The results of the present study indicate that liposomeencapsulation increases the bioavailability of midazolam on oral administration.

Reference 1:

Tomoyasu, Y., Yasuda, T., Maeda, S., Higuchi, H. and Miyawaki, T.: Liposomeencapsulated midazolam for oral administration. J. Liposome Res., 21, 166-172, 2011.

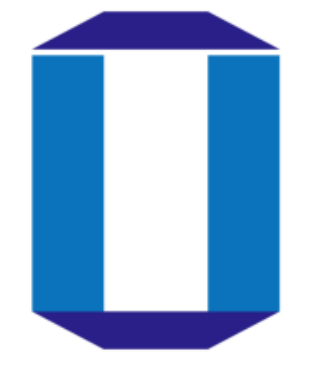

OKAYAMA UNIV. 